\title{
CLOSING COMMENTS: THEORY
}

\author{
C. S. SHUKRE \\ Raman Research Institute
}

Though it had been circulating through the grapevine for more than ten years, it is now widely known that the ion work function on the neutronstar surface is so low that the surface may not even be a solid (Kössl et al. 1988). In this meeting the issue surfaced in the review by Arons, and came up in many discussions by the participants. A view was expressed that since a real "professional" calculation is not yet available, one should reserve judgement on this issue. However, three different methods lead to about the same value, a fraction of a $\mathrm{keV}$, for the ion work function. Furthermore, the polar cap region in pulsars will be hot and will have strong electromagnetic fields. Thus easy extraction of ions from the stellar surface appears most likely.

The Ruderman-Sutherland (RS) model, which was conceded even by its authors not to be the final word on pulsar radiation, is still popular with those who want a framework to analyze pulsar observations. The "user friendly" nature of this model is a virtue not shared by others. Another strange virtue (strange because it does not apply to half of the neutron stars, i.e., the "antipulsars") of this model is a mechanism to produce accelerating voltages in the polar cap regions. Such a mechanism is considered an essential part of any model of pulsar radiation. But the $\mathrm{RS}$ mechanism can work only if ion extraction from the stellar surface is difficult if not impossible. However, with the ion work function being so small we are once again faced with the problem of how to generate this almost uninterrupted power supply.

Among the existing models, only one, i.e. the 'slot gap' modei (Arons 1981c), can still provide it. However, it has not yet been worked out how this model can be matched to global magnetospheric properties. The Beskin, Gurevich, and Istomin (BGI) model, to some extent deals with the global picture, but solves the accelerating voltage problem by assumption. In this respect the poster contribution of Muslimov and Tsygan is very interesting. It would appear that through general relativistic ef- fects alone one can generate such voltages. Most likely this mechanism will turn out to play a complementary role in a slot-gap type model.

A related issue, about which we were supposed to have a poster in the meeting by Shabad and Usov, concerns electron-positron pair creation in neutron-star magnetic fields. It was claimed some time ago that they created pairs from virtual positronium and thus do not produce a cascade. The cascade is another crucial requirement to produce the large particle densities needed. The consensus that has emerged is that this problem, associated with strong magnetic field pulsars, does not conflict with ideas of polar cap models (Bhatia, Chopra, and Panchapakesan 1988). In the region where the cascade is supposed to occur there also exists an electric field with components both perpendicular and parallel to the magnetic field. This will further aid the particle production, which is again something which had been known but is usually forgotten.

Reservations about the instability invoked in the BGI model were expressed by many. Furthermore, as with any other model there are a few other issues to be settled. These have been reviewed by Arons and Melrose. There was very little in the panel discussion which could add to these reviews. In conclusion, the problems with the BGI model look surmountable.

With the magnetic fields of pulsars now alleged to be not decaying, as $\mathrm{McCulloch}$ has pointed out, the problem of nulling needs to be thought about afresh. Can we conclude that the millisecond pulsars-whose magnetic field and period both are almost unevolving-will never null?

\footnotetext{
Acknowledgments: This report summarizes impressions of some issues not covered by other panelists and is based on talks in the Colloquium and ensuing discussions with numerous participants. I want to especially thank J. Arons, A. V. Gurevich and D. B. Melrose for very helpful discussions and R. Romani for comments.
} 